\title{
Photoacoustic generation of focused ultrasonic pulses with predefined temporal profiles including quasi- unipolar pressure pulses
}

Konstantin Maslov, Hao F. Zhang, Lihong V. Wang

Konstantin Maslov, Hao F. Zhang, Lihong V. Wang, "Photoacoustic generation of focused ultrasonic pulses with predefined temporal profiles including quasi-unipolar pressure pulses," Proc. SPIE 6856, Photons Plus Ultrasound: Imaging and Sensing 2008: The Ninth Conference on Biomedical Thermoacoustics, Optoacoustics, and Acousto-optics, 68560C (28 February 2008); doi: 10.1117/12.763972

SPIE. Event: SPIE BiOS, 2008, San Jose, California, United States 


\title{
Photoacoustic Generation of Focused Ultrasonic Pulses with Predefined Temporal Profiles Including Quasi-unipolar Pressure Pulses
}

\author{
Konstantin Maslov ${ }^{\mathrm{a}}$, Hao F. Zhang ${ }^{\mathrm{b}}$, Lihong V. Wang ${ }^{\mathrm{a}}$ \\ a Optical Imaging Laboratory, Department of Biomedical Engineering, \\ Washington University in St. Louis, St. Louis, Missouri 63130 \\ ${ }^{\mathrm{b}}$ Department of Electrical Engineering and Computer Science, \\ University of Wisconsin-Milwaukee, P.O. Box 748 Milwaukee, WI 53201-0784
}

\begin{abstract}
One of the applications of the photoacoustic effect in biomedical research is generation of ultra-short acoustic pressure pulses in tissue. An acoustic wave is generated directly in tissue or in an acoustically well coupled immersion liquid, thus avoiding mechanical resonances of the piezoelectric ultrasonic transducer. Although laser generation of the unipolar pressure pulses has been proposed and used before, little attention was paid to the change of the temporal shape of the pulse when it propagates from a transducer. Here we derive simple mathematical solution which helps to predict the pulse shape in the focal region of the transducer and to experimentally verify theoretical calculations showing generation of short quasi-unipolar pressure pulses.
\end{abstract}

Keywords: photoacoustics, ultrasound modulated optical tomography, bio-effects of ultrasound

\section{INTRODUCTION}

In many cases the formation of a pressure pulse of a predefined temporal shape, and of short unipolar pressure pulses in particularly is desired. The goal is to improve spatial resolution of the ultrasonic nondestructive evaluation and simplify wavelet decomposition of the received ultrasonic signal in ultrasound modulated optical tomography ${ }^{1-3}$ or properly excite certain non-linear effects such as cavitation ${ }^{4,5}$.

The attempt to generate such pulses using an ultrasonic transducer meets several obstacles. If the transducer operates around its resonance frequency, it acts as a band-pass filter, which makes it impossible to obtain unipolar pulses. In another approach ${ }^{6}$, thick piezoelectric crystals have been used, which can lead to unipolar generation. However, it is very difficult to obtain a large pressure amplitude in this a way, due to the high impedance of thick crystals, and to the low piezoelectric coefficient of these materials. The efficiency of this transducer can be increased by using the configuration of the electrodes around the transducer which produces a nonsymmetrical electric field with respect to the different surfaces of the transducer ${ }^{7}$. In this case, it is possible to generate unipolar pulses in a material of the same acoustical impedance as that of the transducer itself, hence impedance mismatch between the immersion liquid or biological tissue and the transducer material gives rise to pulse reverberations. Successful generation of unipolar pressure pulses in narrow field of a transducer of plain geometry was described earlier ${ }^{8}$.

In all the above mentioned cases planar, one-dimensional problem geometry has been considered. However, in many applications of ultrasonic technique the major interest is the focal region of the transducer where concentration of the energy of acoustic waves occurs. Due to the change of the temporal profile of the ultrasonic pulse during wave propagation in three-dimensional space a three-dimensional problem must be considered for unipolar pulse generation in focusing ultrasonic systems. Moreover, as is well known, time averaging of the acoustic pressure in any point of the open space must be equal to zero. Hence production of a truly unipolar pressure pulse is impossible and generation of quasi-unipolar pressure pulses having large amplitude difference between the positive and negative pressure maxima should be considered as a valuable substitute. In the current article we derive simple mathematical solution which helps

Photons Plus Ultrasound: Imaging and Sensing 2008: The Ninth Conference on Biomedical Thermoacoustics, Optoacoustics, and Acousto-optics, edited by Alexander A. Oraevsky, Lihong V. Wang, Proc. of SPIE Vol. 6856, 68560C, (2008) - 1605-7422/08/\$18 - doi: 10.1117/12.763972 
to predict the pulse shape in the focal region of the transducer and to experimentally verify theoretical calculations showing generation of short quasi-unipolar pressure pulses.

\section{THEORY}

\subsection{Sound generation by laser pulse}

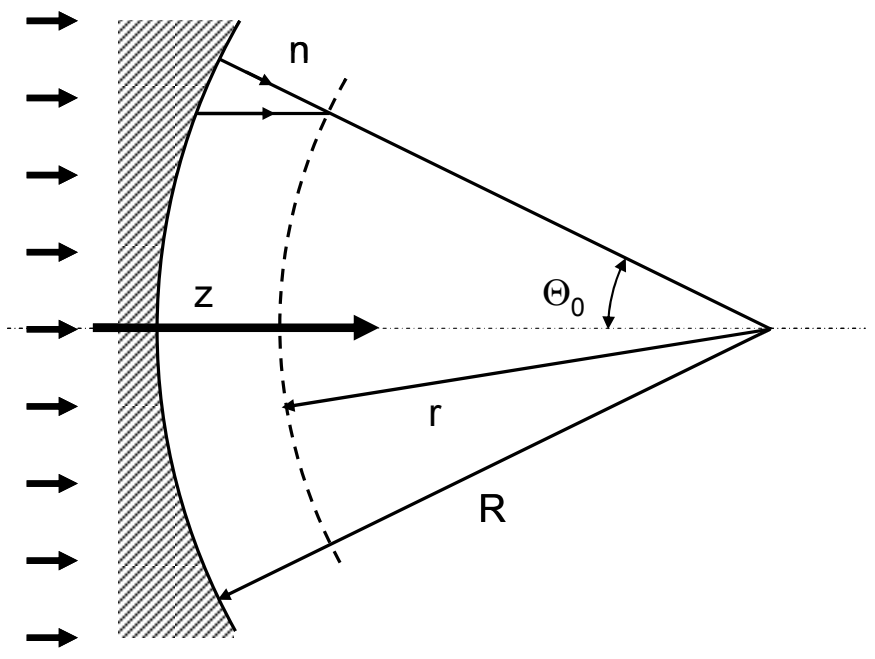

Fig. 1. Problem geometry.

Consider the problem shown in Fig.1. A round laser beam of intensity $I_{0}$ uniformly illuminates an optically absorbing medium through an optically transparent concave window. The spherical cavity has a radius of curvature $R$ and opening angle $\theta_{0}$. The absorption of electromagnetic radiation gives rise to a linear thermal expansion of the media. The motion from thermal expansion produces sound waves ${ }^{9}$. Since acoustic radiation is emitted wherever heat is deposited, the spatial and temporal character of the emitted acoustic wave is defined by the geometry and optical properties of the absorber and the spatial and temporal profile of the laser beam. In a uniformly absorbing medium with an optical absorption coefficient $\mu_{a}$, light intensity exponentially decays in the direction of cavity axis of symmetry, $z$, as $I=I_{0} \exp \left(-\mu_{a} z\right)$. Let us limit consideration to large transducer $(k R>>1$, where $k=\omega / c$ is acoustic wave number) with small numerical aperture, $\sin \theta_{o}<<1$. Let us also assume that optical absorption is relatively high, $\mu_{a} R>>1$. That is reasonable assumption because as it will be shown below and has been already shown elsewhere ${ }^{9}$ efficiency of the photoacoustic sound generation is proportional to $\mu_{a}$. Two requirements namely, $k R>>1$ and $\mu_{a} R>>1$, are actually related to each other because the central frequency of the photoacoustically generated sound wave, $\omega_{0}$, corresponds to the wave number $k_{0} \sim \mu_{a}$. Under these assumptions the area near concave surface can be treated as locally flat, and sound wave generation considered as one dimensional problem in rectangular coordinates.

For a fluid, the coupled differential equations for the pressure and temperature uncouple, giving a heat diffusion equation and a wave equation for pressure. Moreover, when the thermal diffusion length is much less then acoustic wavelength, $\delta_{T}<<\lambda_{S}$, the effect of thermal conductivity on the initial pressure distribution can be neglected. In water, for laser pulses with pulse width $\tau_{H}$, abovementioned heat confinement conditions are

$$
\delta_{T}=\sqrt{K_{T} \tau_{H}}<<\lambda_{s}=c \cdot \tau_{H} \rightarrow \tau_{H}>>10^{-13} s,
$$

where $K_{T}$ is thermal diffusivity and $c$ is speed of sound in the water, which holds in all known ultrasonic applications. 
With the assumption of $\delta_{T}<<\lambda_{s}$, the pressure distribution is given by an inhomogeneous wave equation for sound waves?:

$$
\nabla^{2} p(r, t)-\frac{1}{c^{2}} \frac{\partial^{2}}{\partial t^{2}} p(r, t)=-\frac{\beta}{C_{p}} \frac{\partial}{\partial t} E(r, t),
$$

where $p$ is acoustic pressure, $\beta$ is the isobaric volume expansion coefficient, $C_{p}$ is the specific heat, and $E(r, t)$ is the heat deposition.

The momentary pressure distribution caused by impulse excitation in the one dimensional case is given by ${ }^{8}$ :

$$
p(r)=\frac{\beta c^{2}}{C_{p}} \mu_{a} H(r)=\mu_{a} \Gamma H(r)
$$

where $\Gamma$ is the Gruneisen parameter which in water can be approximately given by the empirical formula ${ }^{8}$ :

$$
\Gamma=0.0043+0.0053 / \mathrm{T}_{C},
$$

where $H(r)$ is the spatial distribution of optical fluence in $\mathrm{J} / \mathrm{cm}^{2}, T_{C}$ is the water temperature in degrees Celsius, pressure is measured in millipascals, and the absorption coefficient of the medium in $\mathrm{cm}^{-1}$.

The initial particle velocity, $v$, is equal to zero, hence d'Alembert's for the problem in hand is a sum of two plane wave traveling in opposite directions:

$$
p(t, z)=\mu_{a} \Gamma[H(t+z / c)-H(t-z / c)] .
$$

Taking into account that $\sin \theta_{o}<<1$ and $\mu_{a} R<<1$ (5) is equivalent to solution in spherical coordinates:

$$
p(t, r)=\mu_{a} \Gamma \frac{R}{r}[H(t+r / c)-H(t-r / c)] .
$$

If $\mu_{a}=$ const, then the pressure on the concave surface in the case of a rigid boundary is given by:

$$
p(t, R)=\mu_{a} \Gamma H_{0} \exp \left(-\mu_{a} c|t|\right) .
$$

In the case of a free boundary, pressure wave reflection occurs with $\pi$ phase shift, which gives the pressure on concave surface as

$$
p(t, R)=\mu_{a} \Gamma H_{0} \exp \left(-\mu_{a} c|t|\right)(1-2 h(t)),
$$

were $h(t)=0 @ t<0$ and $h(t)=1 @ t \geq 0$.

In a one dimensional problem, pressure at any point can be found using d'Alembert's formula from (7), by introducing delay on $t=z / c$. Pressure pulse described by (7) has a familiar shape of a unipolar pulse with pulse width $\tau \approx 1 /\left(\mu_{a} c\right)$ defined by the optical absorption. In the case of a finite size transducer (or laser beam diameter, equivalently) in unbounded medium, solutions (7) and (8) are valid only near the cavity boundary. Moreover, as is well known, the simple integration of the equation of motion

$$
\rho \frac{\partial v}{\partial t}+\nabla p=0
$$

over time and taking into account initial pressure distribution $p(t, \infty)=0$ and assuming zero particle velocity at infinity shows that the time-averaged pressure at any point should be equal to zero and that unipolar pressure pulse simply cannot be generated. Mathematically, 


$$
\int_{-\infty}^{+\infty} p(t, x) d t=0
$$

The solution for ultrasonic pressure generated by a spherical transducer, as derived in classical work of O’Nail ${ }^{10}$, can be approximated in the focal point by:

$$
p(t, 0)=-\frac{R \sin ^{2}\left(\theta_{0} / 2\right)}{c} \frac{\partial p_{0}}{\partial t} .
$$

Moreover, similar to the plane wave solution, particle velocity near the focus remains proportional to the acoustic pressure, at least for ultrasonic frequencies which are not too low $(k R<<1)$ :

$$
v(t, 0)=\frac{p(t, 0)}{\rho c}\left(\frac{\left(1+\cos \theta_{0}\right)}{2}-\frac{\cos ^{2} \theta_{0}}{i k R}\right) \approx \frac{\left(1+\cos \theta_{0}\right)}{2} \frac{p(t, 0)}{\rho c} .
$$

For a finite pulse width of the excitation laser pulse the temporal pressure profile can always be found by convolving the system pulse response given by (7), (8), and (11) with the temporal profile of the laser pulse ${ }^{11}$. In case of the rigid and free boundaries it gives near transducer surface

$$
\begin{gathered}
p(t, R)=\mu_{a} \Gamma H_{0} \exp \left(-\mu_{a} c|t|\right) \otimes H(t), \text { and } \\
p(t, R)=\mu_{a} \Gamma H_{0} \exp \left(-\mu_{a} c|t|\right)(1-2 h(t)) \otimes H(t)
\end{gathered}
$$

respectively. For the temporal pressure profile in focus it becomes:

$$
\begin{aligned}
p(t, 0) & =\frac{R \sin ^{2}\left(\theta_{0} / 2\right)}{c} \mu_{a} \Gamma H_{0} \frac{\partial}{\partial t}\left[\exp \left(-\mu_{a} c|t|\right)(1-2 h(t)) \otimes H(t)\right], \text { and } \\
p(t, 0) & =\frac{R \sin ^{2}\left(\theta_{0} / 2\right)}{c} \mu_{a} \Gamma H_{0} \frac{\partial}{\partial t}\left[\exp \left(-\mu_{a} c|t|\right)(1-2 h(t)) \otimes H(t)\right]= \\
& =\frac{R \sin ^{2}\left(\theta_{0} / 2\right)}{c} \mu_{a} \Gamma H_{0}\left\{\begin{array}{l}
\mu_{a} c \int_{-\infty}^{+\infty} \exp \left(-\mu_{a} c|t-\tau|\right) H(t) d \tau \\
-2 \exp \left(-\mu_{a} c|t|\right) \cdot H(t)
\end{array}\right\}
\end{aligned}
$$

respectively. For rigid boundary and short compare to $1 /\left(\mu_{a} c\right)$ laser pulse maximum pressure in focus is

$$
p(t, 0)_{\max } \approx \mu_{a}^{2} H_{0} \Gamma R \sin ^{2}\left(\theta_{0} / 2\right) \approx p(t, R)_{\max }\left\{\mu_{a} R \sin ^{2}\left(\theta_{0} / 2\right)\right\}
$$

where $p(t, R)_{\max }$ is maximum pressure near the transducer surface and $\mu_{a} R \sin ^{2}\left(\theta_{0} / 2\right)$ focusing transducer amplification. For free boundary solution (16) consists of two additive parts. One is similar to (17) and another is equal to a product of the pulse shape of the photoacoustic wave generated by plane, rigid boundary photoacoustic transducer $(\mathrm{Eq}, 7)$ with and the temporal profile of the laser pulse.

For simplicity we can consider a typical ${ }^{8}$ approximation of the laser pulse shape by Gaussian function:

$$
H(t)=\frac{1}{\sqrt{\pi}} \frac{H_{0}}{\tau_{H}} \exp \left(-\left(\frac{t}{\tau_{H}}\right)^{2}\right) \quad @ \quad \tau_{H} \mu_{a} c<<1 .
$$

In this case maximum positive pressure in transducer focus is approximately equal to that for transducer with rigid boundary, $p(t, 0)_{\max }^{+} \approx p(t, 0)_{\text {max rigid }}$ while negative pressure maximum has $2 /\left(\mu_{a} \tau_{H} c \sqrt{\pi}\right)$ times large amplitude. Consequently, uniformly absorbing medium with free concave boundary under pulse laser excitation produces in focal 
region quasi-unipolar pulse whose negative pressure part follows pulse shape of the laser pulse with magnitude $2 /\left(\mu_{a} \tau_{H} c \sqrt{\pi}\right)$ higher than magnitude of the positive pressure part.

\section{EXPERIMENTAL SETUP}

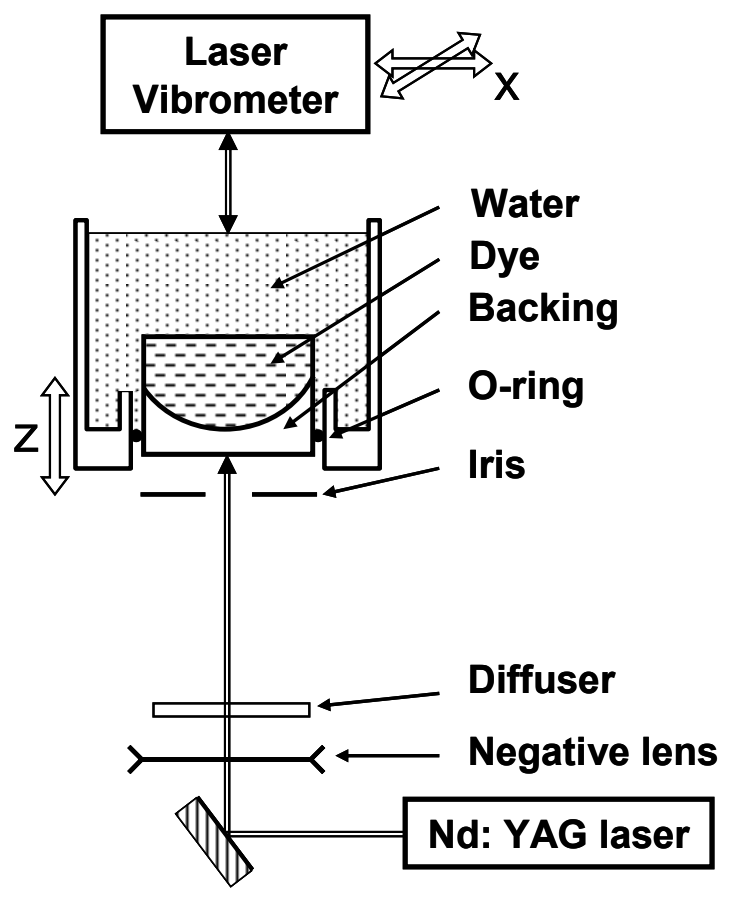

Fig. 2. Experimental setup.

The schematic of the experimental setup is shown in Fig. 2. A Nd:YAG laser (Big Sky) working at low pulse energy generates a laser pulse of $50 \mathrm{~ns}$ duration at $532 \mathrm{~nm}$, with a pulse repetition rate of $10 \mathrm{~Hz}$. The light pulse is expanded by a negative lens and homogenized by a narrow angle ground glass diffuser (DG10-15000, Thorlabs), then shaped by an iris diaphragm. It passes through the backing material into the optically absorbing medium which acts as a photoacoustic transducer. To mimic a rigid boundary, an SF2 glass, plano-concave lens was used as a backing medium. For free boundary conditions, air backing was used. The acoustically matching light absorbing medium was made of a solution of Sudan Red dye in $2 \%$ water-based agar gel. The optical absorption coefficient of the medium was $0.5 \mathrm{~mm}^{-1}$. The rather dense gel maintains its shape in the outer boundary of the photoacoustic transducer and allows using air backing in experiments. A photo of transducers of different shapes is shown on Fig. 3.
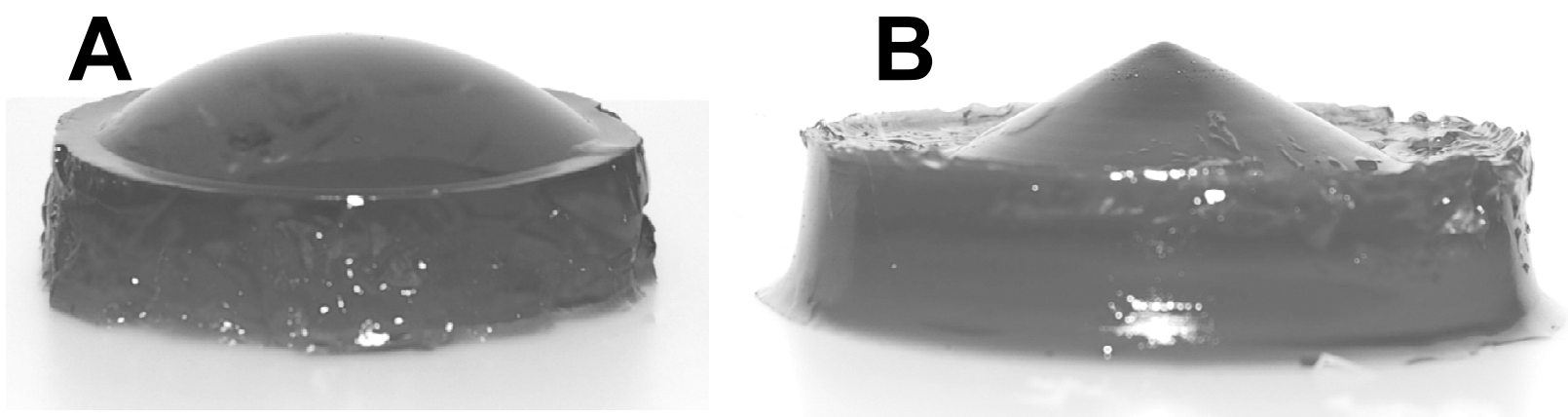

Fig. 3. Photo of the model photoacoustic transducers. (A) Spherically focused transducer; (B) Conical (Bessel focusing) transducer. 
The particle displacement velocity of the ultrasonic pulse was measured on the free surface of the water tank by an OFV 5000 laser vibrometer made by Polytech, equipped with an ODV 505 super heterodyne interferometer head, and VD05 $10 \mathrm{MHz}$ velocity and DD-300 $20 \mathrm{MHz}$ displacement decoders. Vibrometer head were positioned on translational stage to position $50 \mu \mathrm{m}$ diameter measurement point of the vibrometer on transducer axis. Axial positioning was done by changing the water level above the photoacoustic transducer.

\section{EXPERIMENTAL RESULTS}

\subsection{Rigid boundary}
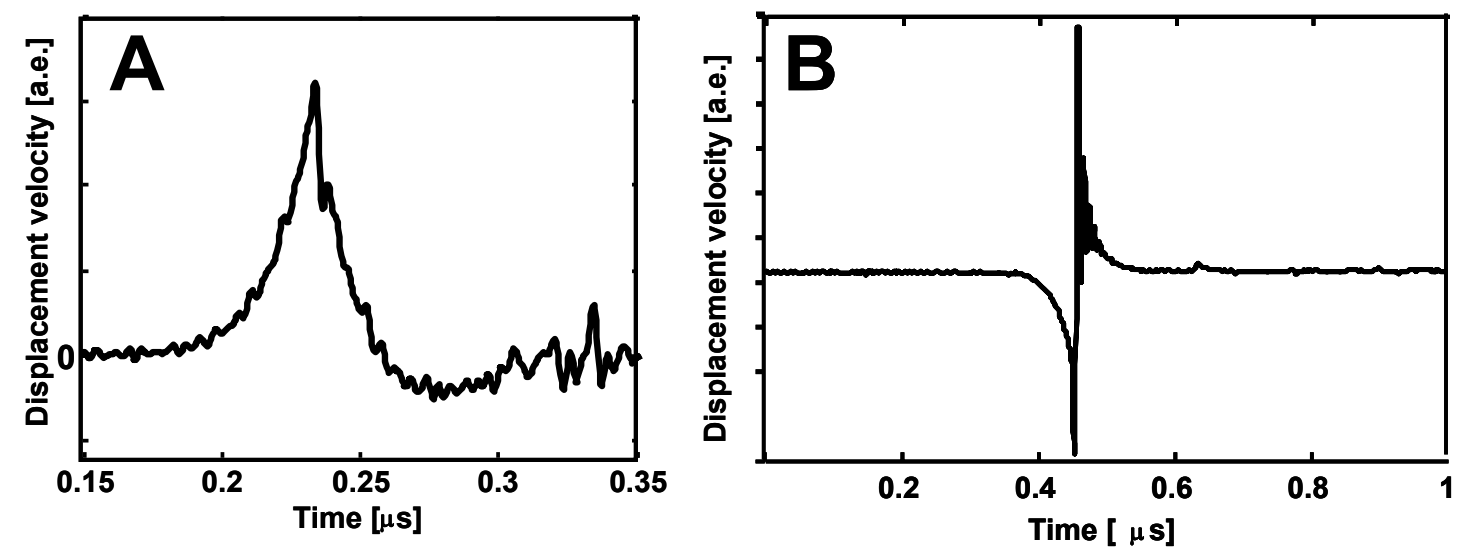

Fig. 4. Temporal profiles of pressure pulses of the photoacoustic transducers with rigid boundaries. (A) Narrow field, plane transducer; (B) Focal region of the spherical transducer.

Temporal profiles of pressure pulses of photoacoustic two different photoacoustic transducers with rigid boundaries, plane and focused, are shown in Fig.4. The signal shown in Fig. 4A has a similar shape to that measured elsewhere ${ }^{8}$. Small oscillations of the signal are noise due to the low amplitude of the unfocused photoacoustic wave. Additional spikes appearing with $0.1 \mu$ s delay are caused by edge effects of the finite size transducer. The photoacoustic signal in focal region of the spherically focused transducer is shown in Fig.4B. In accordance with theoretical calculations it can be well approximated by a time derivative of the signal shown in Fig. 4A. The signal magnitude is much higher than that from the plane transducer due to energy concentration in the focal area. Signal oscillations after a steep change of signal voltage are caused by the finite bandwidth of the vibrometer.

\subsection{Free boundary}
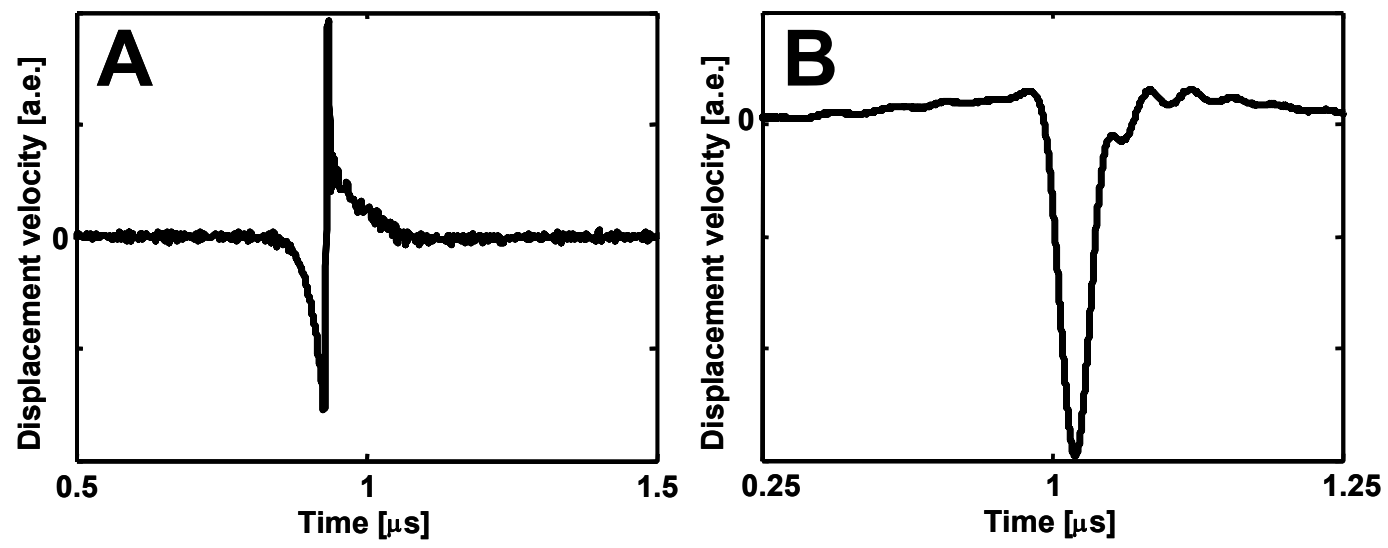

Fig. 5. Temporal profiles of pressure pulses of the photoacoustic transducers with free boundaries. (A) Narrow field, plane geometry; (B) Focal region of the spherical transducer. 
Temporal profiles of the pressure pulses of photoacoustic two different photoacoustic transducers with free boundaries, plane and focused, are shown in Fig.4. Again, the signal shown in Fig. 5A has a similar shape to that measured elsewhere ${ }^{8}$. The photoacoustic signal in the focal region of the spherically focused transducer is shown in Fig.4B. In accordance with theoretical calculations it consists of a superposition of two parts. One part, a positive signal, resembles the signal shown in Fig. 4A. The other part, a negative signal, follows the Gaussian temporal profile of the laser pulse. Good correspondence between theory and experiment, apart from the small oscillations caused by finite bandwidth of the vibrometer, can be noticed.

\subsection{Conical transducer.}

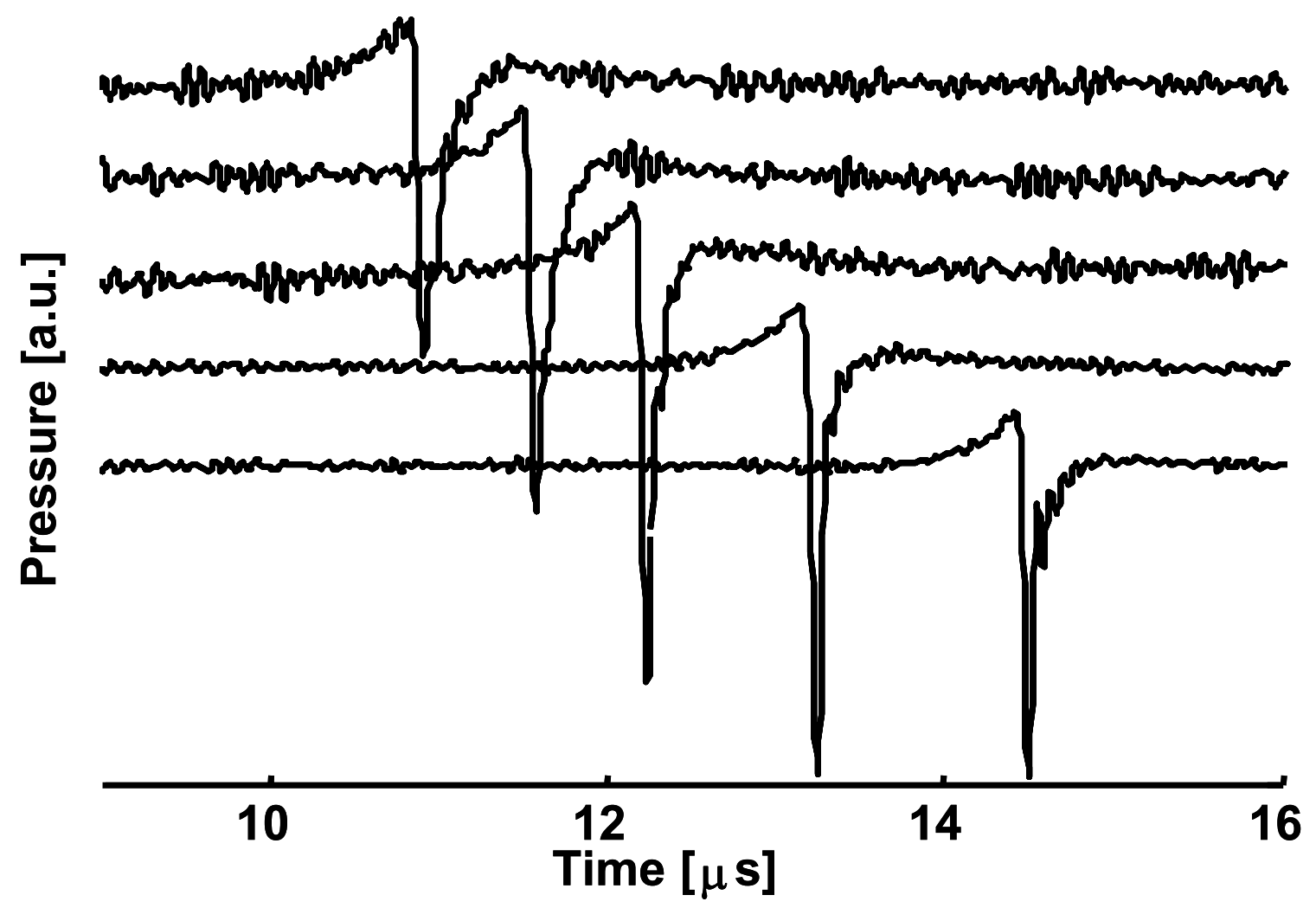

Fig. 6. Temporal profiles of pressure pulses of the conical photoacoustic transducer with free boundary.

Although we were not able to derive a simple solution to predict the photoacoustic pulse shape for any transducer geometry, some features of the pulse shape remain definite for all focusing transducers. One of those features is the strong asymmetry of the photoacoustic pulse in the transducer focal region, which is related to the steep spatial derivative of the initial laser pulse generated pressure distribution on a free boundary of the transducer. As an illustration, temporal profiles of the photoacoustic pressure in the focal region of the conical transducer are shown in Fig. 6. Different curves correspond to different axial positions of the measurement point, as can be seen from the different arrival times of the pressure pulses. As one can see, the pressure pulse preserves its shape when it propagates from the conical transducer. The steep time derivative of the photoacoustic wave on a free surface caused strong asymmetry of the photoacoustic pulse in the focal region, which in this case occupies $6 \mathrm{~mm}$ in length. However, the focusing ability (signal amplification) of the conical transducer is much weaker than that of the spherical transducer, as can be judged from the high noise level of the signals shown in Fig.6. 


\section{CONCLUSION}

For a spherically shaped photoacoustic transducer with a free boundary, a pressure pulse in its focal region can be approximated by the superposition of a positive pressure pulse, which follows the spatial distribution of the optical fluence inside the light absorbing media, and a sharp negative pulse, whose temporal profile follows that of the laser pulse. Because the time-averaged pressure in any case remains zero, the amplitude of the sharp negative pulse is larger than that of the positive pressure pulse by as much as the positive pulse is longer than the negative one. By the regulating absorption coefficient of the transducer material and the width of the laser pulse, it is possible to produce focused, hence space confined, pressure pulses of desired asymmetry.

\section{ACKNOWLEDGEMENT}

This project was sponsored in part by National Institutes of Health Grant Nos. R01 CA106728 and R01 NS46214 (BRP). The authors express special thanks to Mr. Mario Pineda from Polytech, Inc., who made it possible for us to use a laser virbrometer, without which the experimental part of this work could not be performed.

\section{REFERENCES}

[1] S.A. Hariti, S, Hole, and J. Lewiner, "Broadening the potential bandwidth of piezoelectric transducers by partial depolarization," Appl. Phys. Lett., 78, 4037-4039 (2001)

[2] M. DeBilly, L. Adler, and G. Quentin, "About the use of trailing echoes for the detection of disbond at solid-solid interface," J. Phys., 4, 917-921 (1994).

[3] D. A. Sotiropoulos and J. D. Achenbach, "Reflection of elastic-waves by a distribution of coplanar cracks," $J$. Acoust. Soc. Am., 84, 752-759 (1988).

[4] C.C. Church, "A theoretical study of acoustic cavitation produced by "positive-only" and "negative-only" pressure waves in relation to in vivo studies," Ultrasound in Med. \& Biol., 29, 319-330 (2003)

[5] D. Dalecki, et al., "Bioeffects of positive and negative acoustic pressures in mice infused with microbubbles," Ultrasound in Med. \& Biol., 26, 1327-1332 (2000)

[6] W. Eisenmenger and M. Haardt, "Observation of charge compensated polarization zones in polyvinylindenfluoride (PVDF) films by piezoelectric acoustic step-wave response," Solid State Commun., 41, 917-920 (1982).

[7] F. S. Foster and J. W. Hunt, "The design and characterization of short pulse ultrasound transducers," Ultrasonics, 16, 116-122 (1978)

[8] A.A. Oraevsky, S.L. Jacques, and F.K. Tittel, "Measurement of tissue optical properties by time-resolved detection of laser-induced transient stress," Appl. Opt., 36, 402-415 (1997).

[9] I.G. Calasso, W. Craig, and G.J. Diebold, "Photoacoustic point source," Phys. Rev. Let., 86, 3550-3553 (2001)

[10] H. O'Neil, "Theory of focusing radiators," J. Acoust. Soc. Amer., 21, 516-526 (1949)

[11] P.R. Stepanishen, "Transient radiation from pistons in an infinite planar baffle," J. Acoust. Soc. Am. 49, 1629-1638 (1971) 